\title{
A Tissue-Level Electromechanical Model of the Left Ventricle: Application to the Analysis of Intraventricular Pressure
}

\author{
V Le Rolle ${ }^{1,2}$, AI Hernández ${ }^{1,2}$, P-Y Richard ${ }^{3}$, P Pibarot $^{4}$, \\ L-G Durand $^{5}$, G Carrault ${ }^{1,2}$ \\ ${ }^{1}$ INSERM, U642, Rennes, F-35000, France \\ ${ }^{2}$ Université de Rennes 1, LTSI, Rennes, F-35000, France \\ ${ }^{3}$ Supelec IETR, avenue de la Boulaie, BP 81127, 35511 Cesson-Sévigné cedex, France \\ ${ }^{4}$ Institut de cardiologie de Québec, Hôpital Laval, Université Laval, Ste-Foy, Québec, Canada
}

${ }^{5}$ Institut de recherches cliniques de Montréal, Université de Montréal, Montréal, Québec, Canada

\begin{abstract}
This paper presents a model-based method for the analysis of experimental intraventricular pressure. The proposed approach is composed of a ventricular model and an identification algorithm. A multi-formalism, tissue-level electromechanical model of the left ventricle is proposed. Parameter identification is based on evolutionary algorithms and is applied to reproduce experimental pressures obtained from three pigs in steady-state conditions, during afterload variations and during ischemia. Identification results show the capability of the model to reproduce observed intraventricular pressure morphologies and to assist in the interpretation of these signals.
\end{abstract}

\section{Introduction}

The analysis of ventricular pressure measurements is a useful tool for the evaluation of cardiac pumping function. In fact, the morphology of pressure signals reflects the events involved in the cardiac contraction. The modeling of the myocardial behavior can help for the interpretation blood pressure signal morphologies.

Concerning the ventricular function, a variety of mathematical models has been proposed in the literature in order to represent explicitly, at different levels of detail, the cardiac electrical activity [1-3], the excitationcontraction coupling [4-6], the mechanical activity [7,8] and the mechano-hydraulic coupling $[9,10]$. Complete models of the left ventricle result from a combination of these different energy domain descriptions [11,12]. Some studies are based on a fine-grained description of the ventricular activity (at the cellular or sub-cellular levels) $[13,8,14]$. However, these approaches require significant computational resources and are characterized by an important number of parameters. These aspects reduce the model identifiability and makes more difficult to couple these models with models of other physiological systems (e.g. the circulation, autonomic nervous system). On the other hand, the simplest ventricular models are based on a time-varying elastance $[15,16]$, which require low computational resources. However, as the whole left ventricle (LV) is represented with a single element, it is not possible to obtain realistic simulations of cardiac pressure signal morphologies, specially those produced during significant asynchronous activations of different segments of the ventricular wall.

In this paper, a tissue-level electromechanical model of the left ventricle is proposed. Multiple formalisms are employed for the definition of the model. The variety of energy domains, the spatial scale retained (tissue-level) and our will to dispose of a modular model, brought us to use the Bond Graph formalism for the mechano-hydraulic aspects and cellular automata for the electrical activation.

\section{Methods}

The ventricle geometry is assimilated to an ellipsoid composed of twelve coupled mechano-hydraulic subsystems (Figure 1). The asynchronous contraction of these twelve myocardium segments helps to reproduce different pressure signal morphologies.

In order to represent the cardiac electrical activity of each one of the segments, cellular automata are employed. They are characterized by four physiological states [1]: the rapid depolarization period $(R D P)$, the absolute refractory period $(A R P)$, the relative refractory period $(R R P)$ and a waiting period (idle). State transition functions, the sensitivity to heart rate and the interactions between cellular automata are fully described in [17]. 


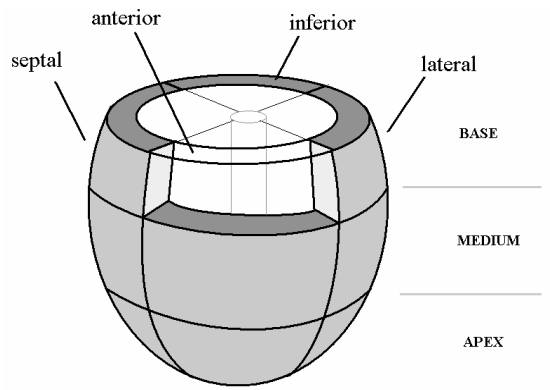

Figure 1. Ventricular model segmentation

The mechanical behavior is described here by the radial force $(F r)$ developed by each wall segment, which is classically represented by the contributions of active and passive properties. The radial force can be computed as the integrated stress over the segment's surface. The total stress is equal to the sum of an active $\left(\sigma_{r_{-} a}\right)$ and a passive stress $\left(\sigma_{r_{-} p}\right)$.

$$
F_{r}=\int \sigma_{r} \cdot d S=\int\left(\sigma_{r_{-} a}+\sigma_{r_{-} p}\right) d S
$$

The active stress tensor can be expressed using the relation:

$$
\sigma_{a}=T_{a} .(F N) .(F N)^{T}
$$

where $F$ is the deformation gradient tensor, $N$ stands for a unitary vector in the fiber direction and $T_{a}$ depends on the intracellular calcium concentration $\left(\left[\mathrm{Ca}^{2+}\right]_{i}\right)$, such that [6]:

$$
T_{a}=T_{r e f}(1+\beta(\lambda-1)) \cdot\left[C a^{2+}\right]_{i}^{n} /\left(\left[C a^{2+}\right]_{i}^{n}+C a_{50}^{n}\right)
$$

where $\lambda$ is the strain, Tref is the value of the tension at $\lambda$ $=1, C a_{50}$ the calcium concentration at $50 \%$ of the isometric tension, $n$ is the Hill coefficient and $\beta$ represents the myofilament "cooperativity". The intracellular calcium concentration is represented in a simple manner as:

$$
\left[\mathrm{Ca}^{2+}\right]_{i}=\left\{\begin{array}{cc}
0 & t_{e s}<0 \\
K \sin \left(\pi \cdot t_{e s} / T_{\max }\right) & 0 \leq t_{e s} \leq T_{\max } \\
0 & t_{e s}>T_{\max }
\end{array}\right.
$$

where tes is the time elapsed since the end of the $R D P$ for each segment $s$, Tmax the activation duration and $K$ the maximum level of calcium concentration.

The passive stress tensor can be expressed using the relation:

$$
\sigma_{p}=-p I+2 F \frac{\partial W}{\partial C} F^{T}
$$

where $W$ is the strain energy function, $p$ stands for the hydrostatic pressure, $I$ is the identity matrix and $C$ is the Cauchy-Green tensor computed from $F: C=F^{T} F$. The selected energy function is the one defined by Humphrey et al. [18], which is defined as:

$$
W=c 1 .(\alpha-1)^{2}-c 2 .(\alpha-1)^{3}+c 3(I 1-3)+c 4(I 1-3)(\alpha-1)+c 5(I 1-
$$

gradient tensor and $\alpha=\sqrt{I 4}$. The radial stress is obtained from a well-known relation, called the Laplace relation, which has been shown to be adequate for the estimation of average longitudinal and latitudinal stresses in ventricular walls in [19]:

$$
-\sigma_{r}=\left(\sigma_{\theta} \times e\right) / R_{p}+\left(\sigma_{\varphi} \times e\right) / R_{m}
$$

The parameter $e$ is the wall thickness, $R_{m}$ and $R_{p}$ stand for the radii of curvature in the meridian and parallel directions. By integrating the Laplace relation, the force developed by the wall segment is defined as:

$$
F_{r}=-\int\left(\left(\sigma_{\theta} \times e\right) / R_{p}+\left(\sigma_{\varphi} \times e\right) / R_{m}\right) d S
$$

where the radial and the azimuthal stress are equal to the sum of active and passive components. These mechanical relations provide the constitutive law of two capacitive elements suitable to model the segment.

The mechano-hydraulic interaction between the myocardial wall and the blood inside the ventricular cavity is then characterized by the coupling relation:

$$
P=-F_{r} / S
$$

where $P$ is the pressure at the wall surface, $F_{r}$ is the radial force developed by the wall segment and $S$ can be easily calculated since the surface is ellipsoidal. This mechanical-hydraulic conversion can be represented by a Bond Graph element called transformer.

The blood behavior inside the cardiac cavity should also be described. Indeed, although the flow $Q$ is supposed to remain the same in each cavity segment, the pressure varies from the wall surface $\left(P_{S}\right)$ to the cavity center $\left(P_{c}\right)$ because of the blood viscosity in order to define an hydraulic resistance $R$ :

$$
Q=\left(P_{c}-P_{s}\right) / R
$$

It is also necessary to take into account the blood mass effects that bring inertial properties $(I)$ :

$$
P_{c}-P_{s}=I d Q / d t
$$

In the Bond Graph formalism, the hydraulic blood activity is modeled by a resistance and an inertance (Figure 2.A). Segments are connected in the hydraulic domain, as the total flow is the sum of the contribution of each segment at the center of each layer. This relation is translated in Bond Graph by a 0-junction (Figure 2.B).
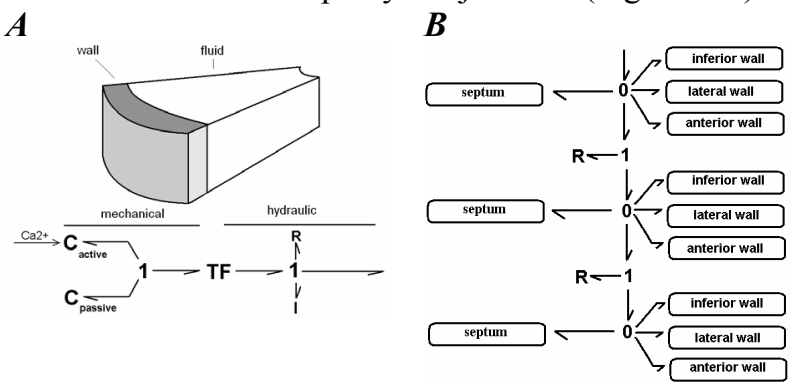

Figure 2. A: Bond Graph model of each mechanohydraulic segment, B: Bond Graph model of the coupled

$I 1$ and $I 4$ stand for the invariants of the deformation 
myocardial segments and a simplified circulatory network.

The parameters values of the mechanical part have been taken from the literature $[5,20]$. The ellipsoid dimensions correspond to mean values measured on healthy adults using Doppler Echography. The other parameters are identified by minimizing the difference between real and simulated ventricular pressure signals using Evolutionary Algorithms (EA). EA have been chosen mainly because it is a pertinent method for the identification of complex nonlinear problems characterized by a poorly-known and high-dimensional state-space [21]. Each individual of the EA represents an instance of the whole model and is characterized by 21 parameters, to be identified: the duration of the depolarization period $(R D P)$, six values for hydraulic resistances and the period $T$ and amplitude $K$ of calcium concentration for each myocardial layer.

The proposed model is used to reproduce and analyze intra-cardiac pressure signals by adapting the model parameters to real data. Experimentations were carried out at the Laval Hospital Research Center, Laval University, Quebec, in 2002 on 3 pigs weighting between 36 and $48 \mathrm{Kg}$. Animal care and experiments were conducted in accordance with the Guidelines of the Canadian Council for Animal Care. The protocol was approved by the Institutional Animal Care Committee of Laval University.

A left lateral thoracotomy was performed before the acquisition. The intra-ventricular pressure is obtained by the insertion of a catheter (Millar Instrument, SPR-350, $5 \mathrm{~F})$ by the right carotid artery. The intra-myocardial pressure acquisition is done by a needle-type catheter (Millar Instrument, SPR-477, 2F) that is inserted inside the myocardial wall. The signals obtained are analysed for different afterload. The afterload augmentation and the afterload diminution are made by the aorta coarctation and by sodium nitroprussiate injection. . The myocardial ischemia was caused by the mechanical obstruction of blood flow in the left descending coronary artery.

\section{Results}

Identification results show the capacity of the proposed model to reproduce different intraventricular pressure morphologies (Figure 3).
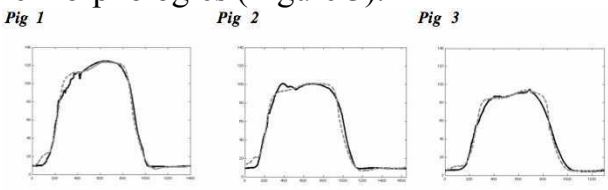

Figure 3. Simulated (black continuous line) and experimental (grey dotted line) ventricular pressure in steady-state conditions.

Besides, electro-mechanical activation times have been determined in the identification process. The maximum electrical activation time is respectively of 101 $\mathrm{ms}, 63 \mathrm{~ms}$ and $75 \mathrm{~ms}$ for the first, second and third pig.

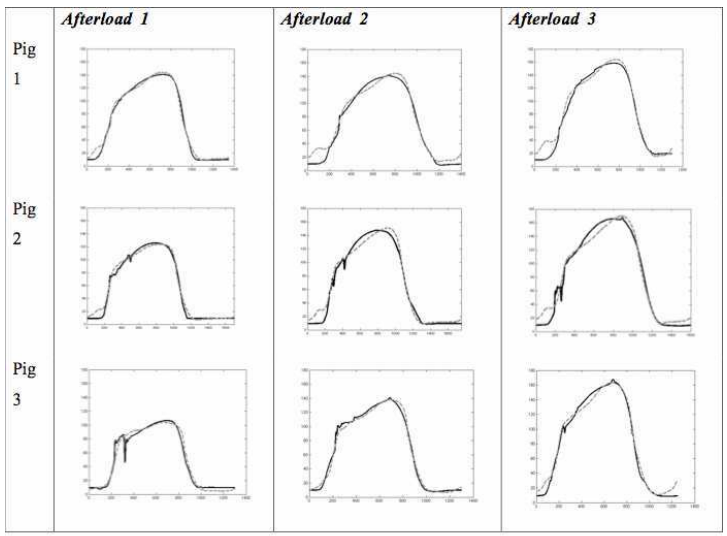

Figure 4. Simulated (black continuous line) and experimental (grey dotted line) ventricular pressure during different afterload conditions.

The alfterload variation case (Figure 4) is particularly interesting, since the aortic resistance augmentation during the afterload rise has been quantified (Figure 5).

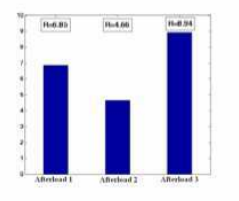

B

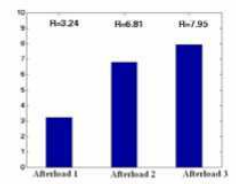

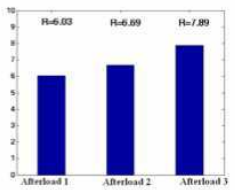

Figure 5. Aortic Resistance value (mmHg.s/ml) for the three afterload conditions.

Finally, the analysis of the identified parameters during ischemia (Figure 6) shows a significant electromechanical desynchronisation. In fact, during ischemia, mechanical activity peak times are respectively equal to $93 \%, 67 \%$ and $65 \%$ of the cardiac cycle duration for the first, second and third pigs.
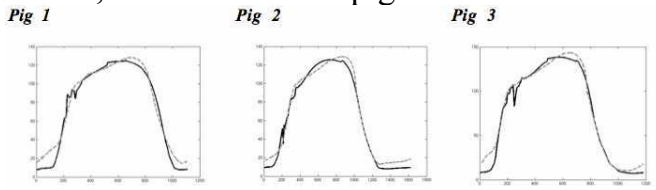

Figure 6. Simulated (black) and experimental (grey) ventricular pressure during ischemia. 


\section{Discussion and conclusions}

The present paper proposes a multiformalism electromechanical model of the left ventricle. An identification algorithm is used in order to define the model parameters in function of experimental ventricular pressures. The simulations, obtained after parameter identification, show the model's capacity to reproduce different morphologies of the intraventricular pressure. This property is mainly due to the model structure that takes into account a simplified geometry, a description of the electrical, mechanical and hydraulic activities and a physiological segmentation. One of the advantages of the approach is the low computational cost that facilitates the parameters identification.

These preliminary results are encouraging for the application of the model on non-invasive data like ECG, arterial pressure or myocardial strain. However, results have to be taken very carefully because the robustness of the identification has not been proved so far. Current work is focused on the improvement of the model and the application of this model-based approach to a concrete clinical problem in the context of heart failure and cardiac resynchronization therapy.

\section{References}

[1] Bardou A. L., Auger P. M., Birkui P. J., and Chasse J. L., Modeling of cardiac electrophysiological mechanisms: from action potential genesis to its propagation in myocardium, Crit Rev Biomed Eng., 1996, 24, 141-221.

[2] FitzHugh RA, Impulses and physiological states in theoretical models of nerve membrane, 1961, 445466.

[3] Luo C. H. and Rudy Y., A dynamic model of the cardiac ventricular action potential. II. Afterdepolarizations, triggered activity, and potentiation, Circ Res., 1994, 74, 1097-113.

[4] Bovendeerd P. H., Borsje P., Arts T., and van De Vosse F. N., Dependence of intramyocardial pressure and coronary flow on ventricular loading and contractility: a model study, Ann Biomed Eng., 2006, 34, 1833-45.

[5] Hunter P. J., McCulloch A. D., and ter Keurs H. E., Modelling the mechanical properties of cardiac muscle, Prog Biophys Mol Biol. 69, 1998, 289-331.

[6] Rice J. J., Jafri M. S., and Winslow R. L., Modeling short-term interval-force relations in cardiac muscle, Am J Physiol Heart Circ Physiol. 2000, 278, H91331 .

[7] Humphrey J. D., Strumpf R. K., and Yin F. C., Determination of a constitutive relation for passive myocardium: II. Parameter estimation, J Biomech Eng. 1990, 112, 340-6.
[8] Nickerson D., Smith N., and Hunter P., New developments in a strongly coupled cardiac electromechanical model, Europace., 2005, 7(2), 118-27.

[9] Chahboune B., J.M. Crolet, Numerical simulation of the blood-wall interaction in the human left ventricle, Eur. Phys.,1998, 2, 291-297.

[10] Kerckhoffs R. C., Bovendeerd P. H., Kotte J. C., Prinzen F. W., Smits K., and Arts T., Homogeneity of cardiac contraction despite physiological asynchrony of depolarization: a model study, Ann Biomed Eng. 2003, 31, 536-47.

[11]Nash M., Mechanics and Material Properties of the Heart using an Anatomically Accurate Mathematical Model. Auckland, 1998.

[12] Sermesant M., Delingette H., and Ayache N., An electromechanical model of the heart for image analysis and simulation, IEEE Trans Med Imaging. 2006, 25, 612-25.

[13] Kerckhoffs R. C., Bovendeerd P. H., Kotte J. C., Prinzen F. W., Smits K., and Arts T., Homogeneity of cardiac contraction despite physiological asynchrony of depolarization: a model study, Ann Biomed Eng. 2003, 536-47.

[14] Ubbink S. W., Bovendeerd P. H., Delhaas T., Arts T., and van de Vosse F. N., Towards model-based analysis of cardiac MR tagging data: relation between left ventricular shear strain and myofiber orientation, Med Image Anal. 2006, 10, 632-41.

[15] Guarini M., Urzua J., Cipriano A., and Gonzalez W., Estimation of cardiac function from computer analysis of the arterial pressure waveform, IEEE Trans Biomed Eng. 1998, 45, 1420-8.

[16] Palladino J. L. and Noordergraaf A., A paradigm for quantifying ventricular contraction, Cell Mol Biol Lett. 2002, 7, 331-5.

[17] Hernandez A.I., Carrault G., Mora F. and Bardou A. Model-based interpretation of cardiac Beats by evolutionary algorthms: signal and model interaction, Artif Intell Med, 2002, 26(3), pp. 211-35

[18] Humphrey J.D., Strumpf R.K. and Yin F.C.P. Determination of a constitutive relation for passive myocardium: II. Parameter estimation, in J. Biomech., 1990, 112, 340-346.

[19] Back L., Left ventricular wall and fluid dynamics of cardiac contraction, Math. Biosci. 1977, 36, 257 297.

[20] Chaudhry, H. R., Bukiet, B. and Findley, T. Stresses and Strains in the Passive Left Ventricle, Journal of Biological Systems, 1996, 4(4) : 535-554

[21] Goldberg D.E., Genetic Algorithms in Search, Optimization and Machine Learning, Reading MA Addison Wesley, 1989 